\title{
Lavendustin B
}

National Cancer Institute

\section{Source}

National Cancer Institute. Lavendustin B. NCI Thesaurus. Code C1616.

A weak tyrosine kinase inhibitor which has been used as a negative control analogue for Lavendustin A. ( $\mathrm{NCl})$ 